\title{
PNPLA3 and TNF- $\alpha$ G238A Genetic Polymorphisms in Egyptian Patients with Different Grades of Severity of NAFLD
}

\author{
Mona A. Hegazy1, Rasha M. Abdel Samie1*', Ahmed Ezzat1, Nagwa Ramadan1, \\ Laila A. Rashed ${ }^{2}$, Abeer M. ElSayed ${ }^{3}$ \\ ${ }^{1}$ Internal Medicine Department, Cairo University, Cairo, Egypt \\ ${ }^{2}$ Medical Biochemistry Department, Cairo University, Cairo, Egypt \\ ${ }^{3}$ Pathology Department, National Cancer Institute, Cairo University, Cairo, Egypt \\ Email:"monahegazy@hotmail.com,drrasha76@gmail.com, ahmedezzatahmed@yahoo.com, \\ dr_nagwa2001@yahoo.com, lailaahmedrashed@gmail.com, abeerelsayed9@gmail.com
}

Received 22 February 2016; accepted 8 March 2016; published 11 March 2016

Copyright (C) 2016 by authors and Scientific Research Publishing Inc.

This work is licensed under the Creative Commons Attribution International License (CC BY).

http://creativecommons.org/licenses/by/4.0/

(c) (i) Open Access

\section{Abstract}

Introduction: There is growing evidence that genetic and environmental factors play an important role in the development and progression of non-alcoholic fatty liver disease (NAFLD). We investigated the association of single nucleotide polymorphism (SNP) rs738409 in PNPLA3 gene and TNF- $\alpha$ G238A polymorphism with the development and severity of NAFLD in an overweight and obese Egyptian population. Material and Methods: 100 overweight and obese patients with NAFLD and 30 control subjects were enrolled. All NAFLD patients underwent a confirmatory biopsy. Laboratory investigations included fasting plasma glucose, kidney and liver function tests, liver enzymes, lipid profile and hepatitis markers. Abdominal ultrasound was performed and all subjects were genotyped for (rs738409) PNPLA3 and (rs361525) TNF- $\alpha$ gene polymorphisms using polymerase chain reaction and restriction fragment length polymorphism (PCR-RFLP). Results: The homozygous GG genotype of the PNPLA3 was most frequent among patients with NASH (26\%) as compared to borderline NASH (20.5\%) and simple steatosis $(20 \%)$. Higher serum levels of transaminases were observed in NAFLD patients and controls who were carriers of the G allele of rs738409, but this was not statistically significant. Regarding the TNF- $\alpha$ G238A SNP; the frequency of the A allele was significantly higher in NAFLD patients $(20 \%)$ compared to controls $(5 \%)(p$ value $=0.006$ ). The highest TNF G allele frequency was observed in the NASH group $(88 \%)$ and this was statistically significant ( $p$ value $=0.009$ ). Conclusion: Our study confirmed the association of the PNPLA3 (rs738409) and TNF- $\alpha$ promoter region G238A polymorphisms with susceptibility to NAFLD and its progression.

"Corresponding author.

How to cite this paper: Hegazy, M.A., Samie, R.M.A., Ezzat, A., Ramadan, N., Rashed, L.A. and ElSayed, A.M. (2016) PNPLA3 and TNF- $\alpha$ G238A Genetic Polymorphisms in Egyptian Patients with Different Grades of Severity of NAFLD. Open Journal of Gastroenterology, 6, 53-64. http://dx.doi.org/10.4236/ojgas.2016.63008 


\section{Keywords}

\section{NAFLD, PNPLA3, TNF- $\alpha$, Polymorphism, NASH}

\section{Introduction}

NAFLD is increasingly recognized as the leading cause of chronic liver disease worldwide [1]. NAFLD encompasses a wide spectrum of varying liver histology ranging from simple steatosis to non-alcoholic steatohepatitis (NASH), often leading to fibrosis and eventually cirrhosis with a high risk of liver failure and hepatocellular carcinoma. There is growing evidence that genetic as well as environmental factors play an important role in the development and progression of NAFLD [2]-[4]. In recent years, genetic determinants of steatosis are being revealed using genome-wide association studies [5] [6]. These studies have identified patain-like phospholipase domain containing3 (PNPLA3) gene, also called adiponutrin, which encodes a 481 amino acid membrane protein localized in the endoplasmic reticulum and at the surface of lipid droplets [5].

The first Genome-wide association (GWA) study identified the PNPLA3 gene polymorphism as a major genetic determinant for the predisposition to NAFLD in Hispanic, African American and European Americans populations according to liver fat content [5], which was subsequently confirmed in Europeans and Asians according to liver biopsy. The association of PNPLA3 gene polymorphisms not only with fatty liver and triglyceride content, but also with histological severity of NAFLD was shown in subsequent studies [7] [8].

The investigation of the potential role of tumor necrosis factor- $\alpha$ (TNF- $\alpha$ ) and other pro-inflammatory cytokines in NASH was first enthused by the obvious similarity between NASH and the effect of these cytokines. The progression of NAFLD from simple steatosis to fibrosis is associated with a significant increase in TNF- $\alpha$ [9] [10].

The TNF- $\alpha$ gene is polymorphic at different positions including-G308A and -G238A. TNF- $\alpha$ polymorphisms have been shown to be strongly associated with the risk of developing NAFLD especially in Chinese population and this was confirmed in a meta-analysis by Wang et al. [11].

In the present study we investigated the association of the SNP rs738409 in the PNPLA3 gene and the TNF- $\alpha$ G238A polymorphism with the development and severity of NAFLD in overweight and obese Egyptian population.

\section{Patients and Methods}

A total of one hundred overweight and obese patients (94 females and 6 males) with NAFLD were enrolled in the study from December 2013 to November 2014. They were prospectively recruited from Gastroenterology and Hepatology outpatient clinic of Kasr El Ainy Hospital. Their age ranged from 29 - 57 years. Thirty healthy, non-obese volunteers who were age- and sex-matched, were included as the control group (all of the control subjects were females). An informed consent was obtained from all participants prior to enrolment. The diagnosis of NAFLD was based on ultrasonographic finding of bright liver, which was defined and graded as: a diffuse hyperechoic echo texture (bright liver) (Grade 1); increased liver echo texture compared to the kidney (Grade 2); vascular blurring (Grade 3) and deep attenuation (Grade 4) [12]. A confirmatory liver biopsy was done after a written consent was obtained. The study was conducted with appropriate approval by the Ethics Committee of Cairo University (N-17-2014) in accordance with the ethical guidelines of the Declaration of Helsinki [13].

Inclusion criteria for NAFLD patients were age above 18 years, overweight or BMI $>25 \mathrm{~kg} / \mathrm{m}^{2}$, and bright liver on abdominal ultrasound with or without elevated liver enzymes. Patients were excluded from the study if one of the following criteria were present: any liver disease other than NAFLD such as hepatitis B or C, autoimmune hepatitis, alpha one antitrypsin deficiency or Wilson's disease, alcohol consumption, history of drug intake (such as use of amiodarone, corticosteroids, tamoxifen, methotrexate, oral contraceptives), pregnancy, diabetes, hypertension, thyroid disease, malignancy and decompensated liver disease. Any subjects with evidence of local or systemic infection on physical examination were excluded from the study. In all controls, the absence of any current or past liver disease was established based on the presence of normal liver function tests and the presence of a normal abdominal ultrasound. 
All subjects included in the study were subjected to detailed history taking, complete clinical examination including anthropometric evaluation (weight, height, waist circumference, and BMI was calculated). BMI between $25-30 \mathrm{~kg} / \mathrm{m}^{2}(<30)$ and $\geq 30 \mathrm{~kg} / \mathrm{m}^{2}$ were defined as overweight and obesity respectively. Laboratory investigations included fasting plasma glucose, liver function tests, serum transaminases (ALT, AST), GGT, urea and creatinine, lipid profile, hepatitis markers including hepatitis B surface antigen and hepatitis C virus antibodies. All the patients as well as the controls were genotyped for Rs738409 PNPLA3 gene and Rs361525 TNF gene polymorphisms by polymerase chain reaction and restriction fragment length polymorphism (PCR/RFLP) using specific primer sequence and restriction enzyme.

Abdominal ultrasonography was performed for all subjects using a Toshiba Apilo XV scanner equipped with a broad band $3.5 \mathrm{MHz}$ curved array probe to assess the presence of liver steatosis (bright liver) and by a single operator to avoid inter-observer variability. Patients were examined after at least 8 hours fasting and were examined in the supine, right and left lateral positions.

Liver biopsies were obtained from all patients with NAFLD (diagnosis based on the finding of bright liver on abdominal ultrasound) after a written consent, using an automated gun device and under complete aseptic precautions. The histological features of the biopsies were graded according to the NAFLD scoring system proposed by the National Institute of Diabetes and Digestive and Kidney Diseases NASH Clinical Research Network and reported as NAFLD activity score (NAS) [14].

Total NAS score represents the sum of scores for steatosis ( 0 - 3), lobular inflammation ( 0 - 3), and ballooning $(0$ - 2), and ranges from 0 - 8. Diagnosis of NAFLD was made first, then NAS was used to grade activity. In patients with NAFLD, NAS score of $\geq 5$ strongly correlated with a diagnosis of "definite NASH", 3 or 4 correlated with "borderline NASH", whereas NAS $\leq 2$ correlated with a diagnosis of "not NASH" [14]. The stage of fibrosis was assessed separately from NAS using a four-point scale: $0=$ no fibrosis; $1=$ mild/moderate zone 3 perisinusoidal fibrosis or portal/periportal fibrosis only; 2 = perisinusoidal and portal/periportal fibrosis; 3 = bridging fibrosis and $4=$ cirrhosis. Significant fibrosis was defined as fibrosis grade $\geq 2$ [15].

\section{DNA Preparation and Genetic Analysis}

The genomic DNA was extracted from whole blood samples using a Qiagen amp DNA mini kit (USA) extraction kit, according to manufacturer instruction. The purity and concentration of DNA was determined using spectrophotometry, quality of DNA was also determined on $0.8 \%$ agarose gel electrophoresis and DNA was stored at $-20^{\circ} \mathrm{C}$ until further analyses.

Amplification was carried out in a final volume of $50 \mu \mathrm{L}$ reaction containing $100 \mathrm{ng}$ genomic DNA, $200 \mathrm{mM}$ each dNTP, $1 \mathrm{mM} \mathrm{MgCl}, 10 \mathrm{mM}$ tris-HCl (pH 8.3), $50 \mathrm{mM} \mathrm{KCl,} \mathrm{0.1 \%} \mathrm{Triton} \mathrm{X-100,} 1.5$ units of Taq DNA polymerase (MBI fermentas, Canada), and $1 \mathrm{mM}$ of each of the primers with the following sequence:

Rs361525 TNF with Forward Primer: 5'AGAAGACCCCCCTCGGAACC-3' and

Reverse Primer: 5'-ATCTGGAGGAAGCGGTAGTG-3' [16] and Rs738409 PNPLA3 gene with Forward primer: 5'-TGGGCCTGAAGTCCGAGGGT-3' and Reverse primer: 5'-CCGACACCAGTGCCCTGCAG-3' [17].

The PCR conditions were as follows: $95^{\circ} \mathrm{C}$ for 5 min, and then 37 cycles of $94^{\circ} \mathrm{C}$ for $30 \mathrm{sec}, 66^{\circ} \mathrm{C}$ for $30 \mathrm{sec}$, and $72^{\circ} \mathrm{C}$ for $40 \mathrm{sec}$ and a final extension step of $72^{\circ} \mathrm{C}$ for 5 minutes. Then PCR products were digested overnight at $65^{\circ} \mathrm{C}$ with Taq1 restriction enzyme and BstF5 I (Fermentas, Canada) for PNPLA3 and TNF alpha respectively. Digested PCR products were subjected to horizontal electrophoresis in $1.5 \%$ ethidium bromidestained agarose gels in $1 \mathrm{X}$ TBE buffer at $120 \mathrm{~V}$ for $1 \mathrm{hr}$ and were visualized using WiseDoc WGD-30 (DAIHAN, Korea).

\section{Statistical Methods}

Data were coded and entered using the statistical package SPSS version 21. Data was summarized using mean, standard deviation, median, minimum and maximum for quantitative variables and frequencies (number of cases) and relative frequencies (percentages) for categorical variables. Comparison of quantitative variables was done using the nonparametric Kruskal-Wallis when comparing more than 2 groups and using the nonparametric Mann-Whitney U test when comparing 2 groups. For comparing categorical data, Chi square $\left(\chi^{2}\right)$ test was performed. Exact test was used instead when the expected frequency was less than 5 . Genotype frequencies were compared between the different study groups using chi-square tests. Odds ratio (OR) with $95 \%$ confidence 
intervals was calculated. P value $<0.05$ was taken as statistically significant [18] [19].

\section{Results}

The demographic, anthropometric and laboratory data of NAFLD patients and their age- and sex-matched controls are shown in Table 1. NAFLD patients showed a statistically significant higher BMI, waist circumference, serum levels of AST and ALT, fasting blood glucose, serum levels of triglycerides, total cholesterol, HDL-C and LDL-C. According to the results of liver biopsy, the NAFLD group was divided into three subgroups by the NAS score: Group 1 (Not NASH or Simple Steatosis); included 10 patients (10\%) (9 females and 1 male) with mean age $45.50 \pm 4.58$ years, Group 2 (Borderline NASH); included 44 patients (44\%) (42 females and 2 males) with mean age $43.09 \pm 7.89$ years, Group 3 (NASH) (3 of them had fibrosis); included 46 patients (46\%) (43 females and 3 males) with a mean age $44.07 \pm 7.07$ years. Comparison of clinical and laboratory characteristics of the three NAFLD subgroups (Table 2), revealed that the Simple Steatosis group showed a statistically significant lower mean ALT level ( $\mathrm{p}$ value $=0.025$ ) as compared to the borderline NASH and NASH groups.

Table 1. Clinical and laboratory data of the NAFLD patients and control participants.

\begin{tabular}{cccc}
\hline Parameters & NAFLD $(\mathrm{N}=100)$ & Control $(\mathrm{N}=30)$ & p value \\
\hline Age (years) & $43.78 \pm 7.23$ & $43.03 \pm 7.07$ & 0.613 \\
BMI $\left(\mathrm{kg} / \mathrm{m}^{2}\right)$ & $34.88 \pm 3.80$ & $22.04 \pm 1.56$ & $<0.001$ \\
Waist Circumference (cm) & $106.66 \pm 15.22$ & $72.53 \pm 4.53$ & $<0.001$ \\
ALT (IU/L) & $32.74 \pm 19.03$ & $16.50 \pm 5.08$ & $<0.001$ \\
AST (IU/L) & $33.15 \pm 20.47$ & $17.23 \pm 4.36$ & $<0.001$ \\
GGT (IU/L) & $42.23 \pm 20.00$ & $31.67 \pm 8.79$ & 0.012 \\
FBS (mg/dl) & $105.96 \pm 15.84$ & $97.50 \pm 8.48$ & 0.001 \\
T-CHOL (mg/dl) & $206.07 \pm 33.05$ & $156.87 \pm 7.65$ & $<0.001$ \\
LDL-C (mg/dl) & $103.41 \pm 19.50$ & $86.57 \pm 6.59$ & $<0.001$ \\
HDL-C (mg/dl) & $46.15 \pm 9.46$ & $51.83 \pm 7.20$ & 0.003 \\
TGs (mg/dl) & $166.63 \pm 39.42$ & $111.97 \pm 15.27$ & $<0.001$ \\
\hline
\end{tabular}

All data are expressed as mean \pm SD. BMI; body mass index, AST: aspartate transaminase, ALT: alanine aminotransferase, GGT: Gamma-glutamyl transpeptidase, FBS: fasting blood sugar, T-CHOL: total cholesterol, LDL-c: low density lipoprotein, HDL-c: high density lipoprotein, TG: triglycerides.

Table 2. Clinical and laboratory data in subgroups of NAFLD patients.

\begin{tabular}{ccccc}
\hline Parameters & $\begin{array}{c}\text { Not NASH } \\
(\mathrm{N}=10)\end{array}$ & $\begin{array}{c}\text { Borderline NASH } \\
(\mathrm{N}=44)\end{array}$ & $\begin{array}{c}\text { NASH } \\
(\mathrm{N}=46)\end{array}$ & p value \\
\hline Age (years) & $45.50 \pm 4.58$ & $43.09 \pm 7.89$ & $44.07 \pm 7.07$ & 0.678 \\
BMI $\left(\mathrm{kg} / \mathrm{m}^{2}\right)$ & $33.31 \pm 2.71$ & $34.76 \pm 4.01$ & $35.33 \pm 3.76$ & 0.252 \\
Waist circumference (cm) & $98.10 \pm 10.82$ & $107.48 \pm 14.96$ & $107.74 \pm 15.94$ & 0.113 \\
ALT (IU/L) & $21.20 \pm 7.39$ & $30.66 \pm 17.20$ & $37.24 \pm 21.18$ & 0.024 \\
AST (IU/L) & $29.00 \pm 8.25$ & $29.43 \pm 16.94$ & $37.61 \pm 24.42$ & 0.384 \\
GGT (IU/L) & $34.80 \pm 19.11$ & $43.66 \pm 18.24$ & $42.48 \pm 21.78$ & 0.352 \\
FBS (mg/dl) & $96.60 \pm 25.26$ & $106.57 \pm 15.25$ & $103.07 \pm 12.98$ & 0.293 \\
T-CHOL (mg/dl) & $215.80 \pm 33.81$ & $209.27 \pm 35.22$ & $200.89 \pm 30.51$ & 0.472 \\
LDL-C (mg/dl) & $101.10 \pm 7.71$ & $100.73 \pm 22.26$ & $106.48 \pm 18.26$ & 0.247 \\
HDL-C (mg/dl) & $50.90 \pm 8.86$ & $46.95 \pm 9.58$ & $44.35 \pm 9.20$ & 0.104 \\
TGs (mg/dl) & $159.40 \pm 42.39$ & $172.48 \pm 36.73$ & $162.61 \pm 41.33$ & 0.596 \\
\hline
\end{tabular}

All data are expressed as mean \pm SD. BMI: body mass index, AST: aspartate transaminase, ALT: alanine aminotransferase, GGT: Gamma-glutamyl transpeptidase, FBS: fasting blood sugar, T-CHOL: total cholesterol, LDL-c: low density lipoprotein, HDL-c: high density lipoprotein, TG: triglycerides. 
We observed a highly statistical significant correlation between the grades of NAFLD activity assessed by NAS scoring of liver biopsy specimens and the grades of severity of steatosis by abdominal ultrasound or by liver biopsy with $\mathrm{p}$ values $<0.001$. Most of the patients with simple steatosis (Not NASH) showed grade 1 steatosis $(60 \%)$ by abdominal ultrasound while a majority of patients with NASH (47.8\%) showed grade 3 steatosis and $6.5 \%$ showed fibrofatty changes. The grades of steatosis by abdominal ultrasound showed a highly significant correlation with the grades of steatosis by liver biopsy ( $p$ value $<0.001$ ).

Table 3 shows the distribution of PNPLA3 genotypes (CC, GG, GC) and TNF genotypes (AA, GA, GG) in NAFLD patients and controls (Figure 1, Figure 2). There was an increased frequency of the PNPLA3 high risk G-allele in patients with NAFLD as compared to the controls but these differences were not significant with OR for PNPLA3 C allele versus G allele $=0.835(95 \%$ CI $(0.468-1.490)$; p value $=0.541)$. The frequency of the TNF A allele was significantly higher in NAFLD patients (20\%) than in controls (5\%) with OR for the TNF G allele versus A allele $=4.750(95 \% \mathrm{CI}=1.414-15.954)$; $\mathrm{p}$ value $=0.006)$.

We divided the NAFLD group into the simple steatosis, borderline NASH and NASH groups and performed case-control association studies of the PNPLA3 rs738409 SNP and TNF G238A SNP polymorphisms. There was an increased frequency of the PNPLA3 GG genotype in the NASH group (26.1\%) as compared to borderline NASH and simple steatosis groups (20.5\% and 20\% respectively) (Table 4, Figure 3). The PNPLA3 highrisk G allele frequency was highest in the NASH group (51.1\%) as compared to simple steatosis and borderline NASH groups ( $50 \%$ and $47.7 \%$ respectively) but these differences were not statistically significant (Table 4).

Table 3. Distribution of frequency of PNPLA3 rs738409 polymorphism and TNF- $\alpha$ G238A polymorphism in NAFLD patients and controls.

\begin{tabular}{|c|c|c|c|c|c|c|c|}
\hline & & \multicolumn{4}{|c|}{ Group } & \multirow{3}{*}{ OR (95\%CI) } & \multirow{3}{*}{$\mathrm{p}$ value } \\
\hline & & \multicolumn{2}{|c|}{ Patients (100) } & \multicolumn{2}{|c|}{ Controls (30) } & & \\
\hline & & Count & $\%$ & Count & $\%$ & & \\
\hline \multirow[t]{4}{*}{ PNPLA3 } & CC & 24 & $24.0 \%$ & 10 & $33.3 \%$ & $0.730(0.238-2.2440$ & 0.583 \\
\hline & GC & 53 & $53.0 \%$ & 13 & $43.3 \%$ & $1.214(0.438-3.514)$ & 0.684 \\
\hline & GG & 23 & $23.0 \%$ & 7 & $23.3 \%$ & \multicolumn{2}{|c|}{ Reference } \\
\hline & $\mathrm{GC}+\mathrm{CC}$ & 77 & $77 \%$ & 23 & $76.7 \%$ & $1.019(0.388-2.676)$ & 0.970 \\
\hline \multirow[t]{4}{*}{ TNF- $\alpha$} & AA & 5 & $5.0 \%$ & 0 & $.0 \%$ & \multicolumn{2}{|c|}{ Reference } \\
\hline & GA & 30 & $30.0 \%$ & 3 & $10.0 \%$ & - & 1 \\
\hline & GG & 65 & $65.0 \%$ & 27 & $90.0 \%$ & - & 0.318 \\
\hline & $\mathrm{GG}+\mathrm{GA}$ & 95 & $95 \%$ & 30 & $100 \%$ & - & 0.589 \\
\hline
\end{tabular}

SNP: single nucleotide polymorphism, PNPLA3: Patain-like phospholipase, rs: reference single nucleotide polymorphism.

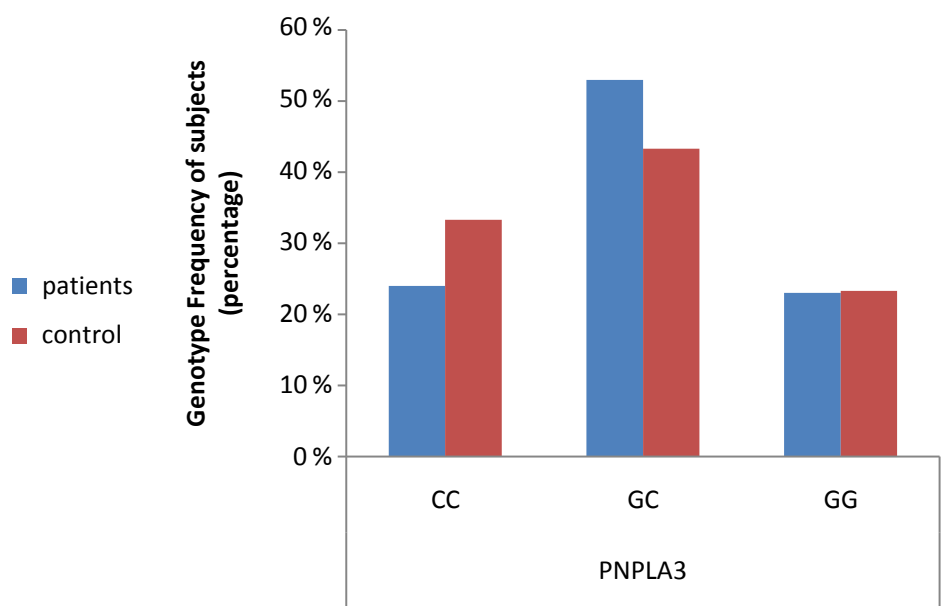

Figure 1. Comparison between PNPLA3 rs738409 SNP in NAFLD patients and controls. 


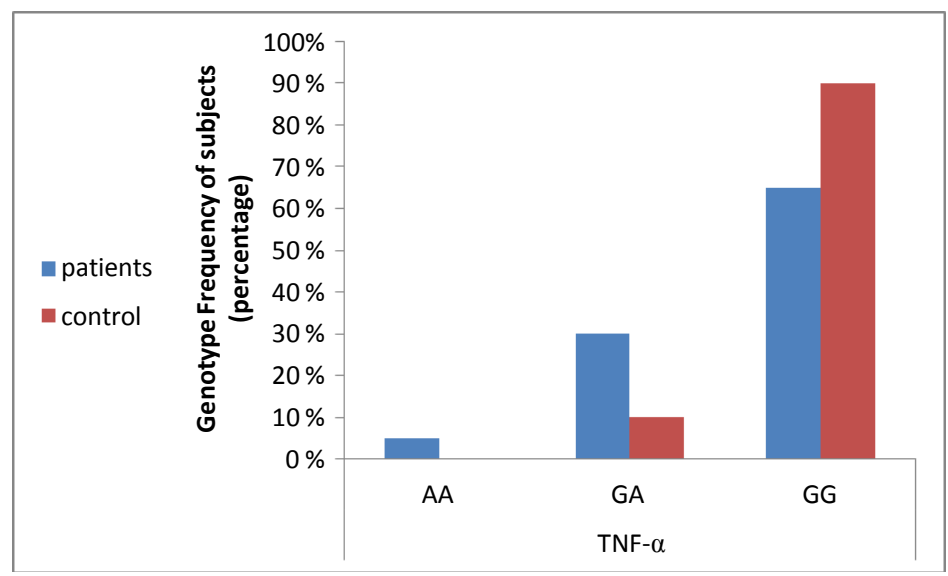

Figure 2. Comparison between TNF- $\alpha$ G238A SNP in NAFLD patients and controls.

Table 4. Distribution of frequency of PNPLA3 rs738409 polymorphism in subgroups of NAFLD patients.

\begin{tabular}{|c|c|c|c|c|c|c|c|c|}
\hline & \multicolumn{8}{|c|}{ Patients subgroups } \\
\hline & & \multicolumn{2}{|c|}{ Not NASH (N. 10) } & \multicolumn{2}{|c|}{ Borderline NASH (N. 44) } & \multicolumn{2}{|c|}{ NASH (N. 46) } & \multirow[t]{2}{*}{$\mathrm{p}$ value } \\
\hline & & Count & $\%$ & Count & $\%$ & Count & $\%$ & \\
\hline \multirow{6}{*}{ PNPLA3 } & CC & 2 & $20.0 \%$ & 11 & $25.0 \%$ & 11 & $23.9 \%$ & \multirow{4}{*}{0.967} \\
\hline & GC & 6 & $60.0 \%$ & 24 & $54.5 \%$ & 23 & $50.0 \%$ & \\
\hline & GG & 2 & $20.0 \%$ & 9 & $20.5 \%$ & 12 & $26.1 \%$ & \\
\hline & $\mathrm{GC}+\mathrm{CC}$ & 8 & $80.0 \%$ & 35 & $79.5 \%$ & 34 & $73.9 \%$ & \\
\hline & Allele C & 10 & $50 \%$ & 46 & $52.3 \%$ & 45 & $48.9 \%$ & \multirow{2}{*}{0.652} \\
\hline & Allele G & 10 & $50 \%$ & 42 & $47.7 \%$ & 47 & $51.1 \%$ & \\
\hline
\end{tabular}

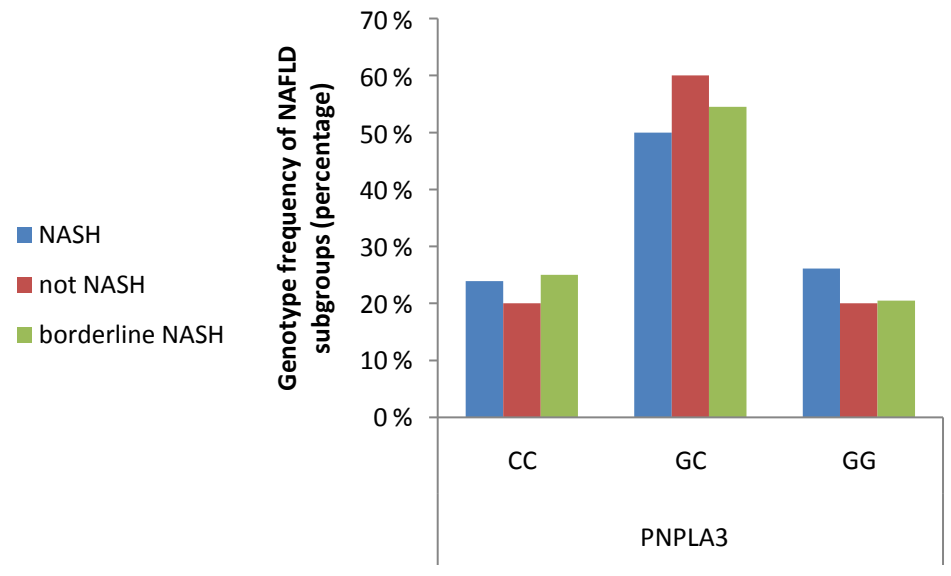

Figure 3. PNLA3 rs738409 SNP results in NAFLD patient's subgroups.

We also observed an increased frequency of the TNF GG genotype in the NASH group (76.1\%) as compared to borderline NASH and simple steatosis groups (56.8\% and 50\% respectively) and these differences were statistically significant $(\mathrm{p}=0.043)$ (Table 5, Figure 4). When comparing the TNF-238 allele frequency among the NAFLD subgroups (Table 5), the highest frequency of the G allele was observed in the NASH group (88\%) and the highest frequency of the A allele was observed in the patients with borderline NASH (27.3\%) and this was statistically significant ( $\mathrm{p}$ value $=0.009$ ). 
Table 5. Distribution of frequency of the TNF- $\alpha$ G238A SNP in subgroups of NAFLD patients.

\begin{tabular}{|c|c|c|c|c|c|c|c|c|}
\hline & \multicolumn{8}{|c|}{ Patients subgroups } \\
\hline & & \multicolumn{2}{|c|}{ Not NASH (N.10) } & \multicolumn{2}{|c|}{ Borderline NASH (N.44) } & \multicolumn{2}{|c|}{ NASH (N.46) } & \multirow[t]{2}{*}{$\mathrm{p}$ value } \\
\hline & & Count & $\%$ & Count & $\%$ & Count & $\%$ & \\
\hline \multirow{6}{*}{ TNF- $\alpha$} & AA & 0 & $0.0 \%$ & 5 & $11.4 \%$ & 0 & $.0 \%$ & \multirow{4}{*}{0.043} \\
\hline & GA & 5 & $50.0 \%$ & 14 & $31.8 \%$ & 11 & $23.9 \%$ & \\
\hline & GG & 5 & $50.0 \%$ & 25 & $56.8 \%$ & 35 & $76.1 \%$ & \\
\hline & $\mathrm{GG}+\mathrm{GA}$ & 10 & $100 \%$ & 39 & $88.6 \%$ & 46 & $100 \%$ & \\
\hline & Allele A & 5 & $25 \%$ & 24 & $27.3 \%$ & 11 & $12 \%$ & \multirow{2}{*}{0.009} \\
\hline & Allele G & 15 & $75 \%$ & 64 & $72.7 \%$ & 81 & $88 \%$ & \\
\hline
\end{tabular}

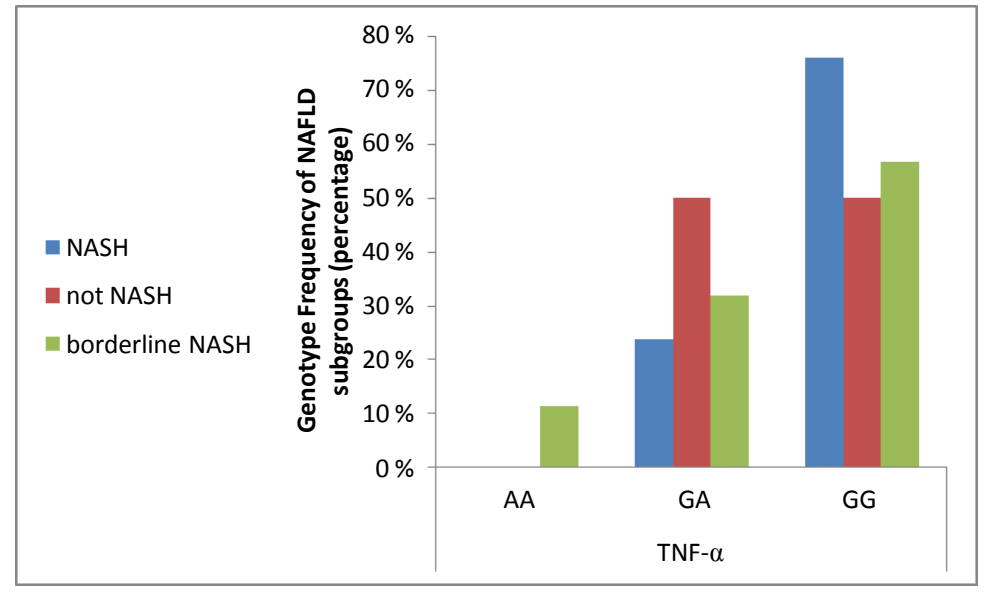

Figure 4. Distribution of the TNF- $\alpha$ G238A SNP in subgroups of NAFLD patients.

Comparison of various quantitative phenotypes (clinical and laboratory parameters) among the different genotypes at rs738409 in PNPLA3 in patients with NAFLD and control subjects showed that the age was significantly higher in NAFLD patients harbouring the risk allele (G-allele) (p value $=0.03$ ). No significant differences in serum levels of AST, ALT, fasting plasma glucose, triglycerides, were observed between patients with NAFLD harbouring the risk allele (G-allele) and those with the homozygous CC genotype. Serum levels of ALT and AST were higher in the control subjects harbouring the risk allele, but these differences were also not significant.

Association of various quantitative phenotypes with the TNF G238A SNP in patients with NAFLD and controls showed that the serum ALT and AST levels were non-significantly higher in patients with NAFLD harbouring the TNF-238 G allele. The serum triglycerides, total cholesterol and LDL-C levels were also higher in NAFLD patients with the G allele, but these differences were also not significant. The serum alkaline phosphatase levels were significantly higher among the control subjects heterozygous for the TNF-238 G allele compared to the homozygous with p value $=0.027$, however no other significant differences were observed.

\section{Discussion}

NAFLD is now considered the leading cause of liver disease worldwide, affecting up to $20 \%$ - $35 \%$ of the general population and is increasingly recognized as a major health concern [20] [21]. In recent years there has been increasing evidence that genetic [5] [22] as well as environmental factors are implicated in the progression of NAFLD [23].

In the present study we evaluated the clinical and metabolic parameters, the liver histology, and the presence 
of polymorphisms in the PNPLA3 gene and the TNF- $\alpha$ promoter region (G-238A) in NAFLD patients and controls. We also investigated the association of these polymorphisms with the progression of NAFLD and their influence on plasma levels of liver enzymes.

Though previous studies have concluded that NAFLD and its complications are more prevalent in women [24] [25], two Indian studies have reported an increased prevalence of NAFLD amongst males [26] [27]. In the current study, $94 \%$ of the studied patients with NAFLD were females and only $6 \%$ were males and so we could not predict any significant gender differences in the prevalence of NAFLD due to the unequal distribution.

The BMI and the waist circumference were found to be significantly higher in NAFLD patients compared to the controls with p value $<0.001$. Moreover, the BMI was found to be the lowest in patients with simple steatosis $\left(33.31 \pm 2.71 \mathrm{~kg} / \mathrm{m}^{2}\right)$ and the highest in patients with NASH $\left(35.33 \pm 3.76 \mathrm{~kg} / \mathrm{m}^{2}\right)$ but these differences in the BMI amongst the different NAFLD subgroups did not reach statistical significance. This agrees with Dixon et al. who found that NAFLD had complicated $94 \%$ of cases with BMI above $35 \mathrm{~kg} / \mathrm{m}^{2}$ [28] and also agrees with the study by Marchesini et al. which confirmed that the presence of metabolic syndrome carried a high risk of NASH among NAFLD subjects [29]. Although a study by Harnois et al. concluded that BMI was the only predictive factor for NASH [30], other studies reported that NAFLD can occur in non obese subjects who are physically inactive [31].

The present study confirmed the finding of an association of the PNPLA3 rs738409 G allele with the susceptibility to NAFLD and its progression. This is in accordance with the results of many trials which showed an association between the rs738409 polymorphism and the risk of NAFLD [32]-[34]. The exact mechanisms by which the I148M polymorphism increases the susceptibility to NAFLD and its influence on the histological severity of NAFLD still remains to be fully elucidated [35]. The homozygous GG genotype was most frequent among the patients with NASH (26\%) when compared to subjects with borderline NASH (20.5\%) and those with simple steatosis (20\%). This was concordant to the results of the meta-analysis by Sookoian and Pirola which showed that the rs738409 exerts a strong influence on the susceptibility to a more aggressive disease with greater hepatic injury, as the GG homozygous subjects had a 3.24-fold more risk of higher necro-inflammatory scores when compared with those who had the CC genotype [8]. Also a meta-analysis by Xu et al. reported that the rs738409 polymorphism was more likely to increase the risk of NASH instead of simple steatosis [35]. Zain and Mohamed, demonstrated a significant association between the GG genotype and the fibrosis stage $(\mathrm{p}=$ 0.038); patients with GG genotype had a greater mean score of 2.03 compared to those with the CC genotype who had a mean score of 1.52. This association between the risk allele $\mathrm{G}$ of the rs738409 and the fibrosis stage, however, could not be assessed in the present study because only 3 patients with NASH had fibrosis [36].

The results of the present study indicated that carriers of the $G$ allele had higher serum levels of transaminases (ALT and AST) and this was observed in the NAFLD patients and control subjects, although this did not reach statistical significance. There have been conflicting reports on the association of the G-allele of rs738409 with the serum transaminases in several studies [5] [7] [37] [38]. In Finnish population with NAFLD, the G allele was found to be significantly associated with increased AST levels, however in Hispanics [5] and in an Italian population [32], it was associated with increased serum ALT levels only. Increased serum levels of both ALT and AST were observed in various populations including the Argentinian [7], Italian [39], Japanese [37] and the Taiwanese [38]. Many factors such as ethnicity, the age group, presence of obesity and/or diabetes could have attributed to the differences in these findings in the various studies. On the contrary, the rs738409 variant was not found to be associated with increased liver enzyme levels (particularly ALT) in various studies conducted on paediatric patients with NAFLD, proven by liver biopsy [40].

In the present study, no significant association between the $G$ allele of rs738409 and the serum triglycerides levels was observed; NAFLD patients harbouring the G allele showed higher level triglycerides as compared to those with the CC genotype, however, decreased serum triglycerides level was observed in the control subjects with GG genotype. This finding does not agree with the findings of Speliotes et al. and Hotta et al. who reported that the G allele of the rs738409 was significantly associated with decreased triglycerides levels in NAFLD patients but not in the control subjects and that this may be explained by decreased production of triglycerides due to the severity of NASH fibrosis [37] [41]. Moreover, in a study by Zain and Mohammed, the triglycerides levels and the $\mathrm{G}$ allele frequency were found to be higher in NASH patients without significant fibrosis compared to NASH patients with significant fibrosis [36]. The variation in our study can thus be explained by the fact that only 3 out of 46 patients with NASH had significant fibrosis and therefore strongly supports the suggestion by Jou et al. that the triglycerides level drops with the extent of hepatocellular injury [42]. 
There have been many conflicting reports on the frequency of G238A and G308A polymorphisms in patients with NASH and controls. Valenti et al. reported a high frequency of G-238A but not of the G-308A TNF- $\alpha$ polymorphisms in Italian patients with NAFLD [43]. On the other hand, Tokushige et al. reported that there were no significant differences in the genotype frequency of any of the five studied TNF- $\alpha$ polymorphisms in Japanese patients with NAFLD as compared to healthy volunteers [44].

To the best of our knowledge, this study is the first to evaluate the presence of polymorphisms in the TNF alpha promoter region at position-238 in Egyptian patients with NAFLD. There was a significant difference in the allele frequency between NAFLD patients and controls for this locus; the frequency of the A allele was higher in patients with NAFLD (20\%) compared to the controls $(5 \%)$ with p value $=0.006$. A study by Yang et al. conducted on paediatric population with NAFLD also confirmed our finding of a higher frequency of the TNF-238 A allele among NAFLD patients as compared to the control subjects, although this difference was not statistically significant [45]. In contrast, Chowdhury et al. did not find any difference in the allele frequency of the TNF $\alpha$-promoter region at position-238 between patients with NAFLD and the control subjects [46]. When analyzing the NAFLD subgroups, we observed an increased frequency of the TNF GG genotype in the NASH group (76.1\%) as compared to borderline NASH and simple steatosis groups (56.8\% and 50\% respectively) but these differences were not statistically significant. When comparing the TNF G allele frequency among the NAFLD subgroups, the highest frequency was observed in the NASH group (88\%) and this was statistically significant with a p value $=0.009$.

We observed an increased frequency of the TNF GA genotype in NASH patients as compared to controls (23.9\% vs $10 \%$ ) but these differences were not significant with p value $=0.126$ for the GA versus the GG genotypes. Our results agree with the results of Trujillo-Murillo et al., who reported that the frequency of the -238 TNF- $\alpha$ polymorphism was significantly higher in NASH patients than in controls $(29 \%$ vs $12 \%$; $\mathrm{p}$ value $=$ 0.0047) [47]. The study by Valenti et al. also found a higher frequency of -238 polymorphisms but not of the -308 TNF polymorphisms in Italian patients with NAFLD [43]. However, a Japanese study by Tokushige et al. did not find any significant differences in the frequency of the -238 TNF- $\alpha$ polymorphisms among NAFLD patients and controls [44]. Although it still remains unclear whether the TNF- $\alpha$ promoter region polymorphisms affect the development of NAFLD, our results suggest that the TNF alpha G238A polymorphism could be associated with NASH.

When analyzing the association of TNF alpha G238A polymorphism with the clinical and laboratory parameters, NAFLD patients with TNF-238 GA genotype showed higher serum triglycerides levels than those with the GG genotype but this did not reach statistical significance. This is in accordance with the study by Cheng et al. conducted to evaluate the association of TNF- $\alpha$ polymorphisms with the risk of developing coronary artery disease (CAD) in NAFLD patients, which also reported that NAFLD patients without CAD who were carriers of the GA genotype had higher serum triglycerides levels compared to those with the GG genotype $(p=0.017)$ [48].

Finally, the present study had several points of strength; first the progression from simple steatosis to NASH did not take a long time duration as was assumed before (age of all patients was almost the same) so it must be searched for in the middle-aged group. Second, this study suggests that genetic variations between individuals can be a strong predictor of disease progression and lastly NASH is a cornerstone of the metabolic syndrome. Some of the limitations of the current study were the predominance of the female gender in the studied groups; unequal distribution of patients in NAFLD subgroups and some statistical obstacles.

\section{Acknowledgements}

We thank Dr. Mohamed Eshra for his help in statistical calculations and we also thank all the participants in our study for their valuable contribution.

\section{Recommendations}

We recommend a future large scale study with an equal gender distribution and an equal distribution of patients within the NAFLD subgroups, as well as regular follow up of patients proven to have NAFLD for early detection of the more aggressive form with NASH and even fibrosis.

\section{Conflict of Interest}

The authors declare no conflict of interest. 


\section{References}

[1] Marchesini, G., Brizi, M., Bianchi, G., et al. (2001) Nonalcoholic Fatty Liver Disease: A Feature of the Metabolic Syndrome. Diabetes, 50, 1844-1850. http://dx.doi.org/10.2337/diabetes.50.8.1844

[2] Gambino, R., Cassader, M., Pagano, G., Durazzo, M. and Musso, G. (2007) Polymorphism in Microsomal Triglyceride Transfer Protein: A Link between Liver Disease and Atherogenic Postprandial Lipid Profile in NASH? Hepatology, 45, 1097-1107. http://dx.doi.org/10.1002/hep.21631

[3] Tokushige, K., Takakura, M., Tsuchiya-Matsushita, N., Taniai, M., Hashimoto, E. and Shiratori, K. (2007) Influence of TNF Gene Polymorphisms in Japanese Patients with NASH and Simple Steatosis. Journal of Hepatology, 46, 11041110. http://dx.doi.org/10.1016/j.jhep.2007.01.028

[4] Wilfred de Alwis, N.M. and Day, C.P. (2008) Genes and Nonalcoholic Fatty Liver Disease. Current Diabetes Reports, 8, 156-163. http://dx.doi.org/10.1007/s11892-008-0027-9

[5] Romeo, S., Kozlitina, J., Xing, C., et al. (2008) Genetic Variation in PNPLA3 Confers Susceptibility to Nonalcoholic Fatty Liver Disease. Nature Genetics, 40, 1461-1465. http://dx.doi.org/10.1038/ng.257

[6] Kamatani, Y., Matsuda, K., Okada, Y., et al. (2010) Genome-Wide Association Study of Hematological and Biochemical Traits in a Japanese Population. Nature Genetics, 42, 210-215. http://dx.doi.org/10.1038/ng.531

[7] Sookoian, S., Castano, G.O., Burgueno, A.L., Gianotti, T.F., Rosselli, M.S. and Pirola, C.J. (2009) A Nonsynonymous Gene Variant in the Adiponutrin Gene Is Associated with Nonalcoholic Fatty Liver Disease Severity. The Journal of Lipid Research, 50, 2111-2116. http://dx.doi.org/10.1194/jlr.P900013-JLR200

[8] Sookoian, S. and Pirola, C.J. (2011) Metabolic Syndrome: From the Genetics to the Pathophysiology. Current Hypertension Reports, 13, 149-157. http://dx.doi.org/10.1007/s11906-010-0164-9

[9] Bayley, J.P., Ottenhoff, T.H. and Verweij, C.L. (2004) Is There a Future for TNF Promoter Polymorphisms? Genes and Immunity, 5, 315-329. http://dx.doi.org/10.1038/sj.gene.6364055

[10] Elahi, M.M., Asotra, K., Matata, B.M. and Mastana, S.S. (2009) Tumor Necrosis Factor Alpha-308 Gene Locus Promoter Polymorphism: An Analysis of Association with Health and Disease. Biochimica et Biophysica Acta, 1792, 163172. http://dx.doi.org/10.1016/j.bbadis.2009.01.007

[11] Wang, J.K., Feng, Z.W., Li, Y.C., Li, Q.Y. and Tao, X.Y. (2012) Association of Tumor Necrosis Factor-Alpha Gene Promoter Polymorphism at Sites-308 and -238 with Non-Alcoholic Fatty Liver Disease: A Meta-Analysis. Journal of Gastroenterology and Hepatology, 27, 670-676. http://dx.doi.org/10.1111/j.1440-1746.2011.06978.x

[12] Ricci, C., Longo, R., Gioulis, E., et al. (1997) Noninvasive in Vivo Quantitative Assessment of Fat Content in Human Liver. Journal of Hepatology, 27, 108-113. http://dx.doi.org/10.1016/S0168-8278(97)80288-7

[13] World Medical Association (2013) Declaration of Helsinki: Ethical Principles for Medical Research Involving Human Subjects. JAMA, 310, 2191-2194. http://dx.doi.org/10.1001/jama.2013.281053

[14] Kleiner, D.E., Brunt, E.M., Van Natta, M., et al. (2005) Design and Validation of a Histological Scoring System for Nonalcoholic Fatty Liver Disease. Hepatology, 41, 1313-1321. http://dx.doi.org/10.1002/hep.20701

[15] Brunt, E.M., Kleiner, D.E., Wilson, L.A., et al. (2009) NASH Clinical Research Network. Portal Chronic Inflammation in Nonalcoholic Fatty Liver Disease (NAFLD): A Histologic Marker of Advanced NAFLD-Clinicopathologic Correlations from the Nonalcoholic Steatohepatitis Clinical Research Network. Hepatology, 49, 809-820. http://dx.doi.org/10.1002/hep.22724

[16] Hedayati, K., Sharifi, F., Rostami, M.S., et al. (2012) Association between TNF-a Promoter G-308A and G-238A Polymorphisms and Obesity. Molecular Biology Reports, 39, 825-829. http://dx.doi.org/10.1007/s11033-011-0804-4

[17] Dutta, A.K. (2011) A New PCR-RFLP Method for Diagnosing PNPLA3 rs738409 Polymorphism. Webmed Central Genetics, 2, 11.

[18] Chan, Y.H. (2003) Biostatistics 102: Quantitative Data-Parametric \& Non-Parametric Tests. Singapore Medical Journal, 44, 391-396.

[19] Chan, Y.H. (2003) Biostatistics 103: Qualitative Data-Tests of Independence. Singapore Medical Journal, 44, 498503.

[20] Angulo, P. (2002) Nonalcoholic Fatty Liver Disease. The New England Journal of Medicine, 18, 1221-1231. http://dx.doi.org/10.1056/NEJMra011775

[21] Farrell, G.C. (2003) Non-Alcoholic Steatohepatitis: What Is It, and Why Is It Important in the Asia-Pacific Region? Journal of Gastroenterology and Hepatology, 18, 124-138. http://dx.doi.org/10.1046/j.1440-1746.2003.02989.x

[22] Wilfred de Alwis, N.M. and Day, C.P. (2007) Genetics of Alcoholic Liver Disease and Nonalcoholic Fatty Liver Disease. Seminars in Liver Disease, 27, 44-54. http://dx.doi.org/10.1055/s-2006-960170

[23] Day, C.P. and James, O.F. (1998) Steatohepatitis: A Tale of Two “Hits”? Gastroenterology, 114, 842-845. http://dx.doi.org/10.1016/S0016-5085(98)70599-2 
[24] Kim, H.J., Kim, H.D., Lee, K.E., et al. (2004) Metabolic Significance of Nonalcoholic Fatty Liver Disease in Nonobese, Nondiabetic Adults. Archives of Internal Medicine, 164, 2169-2175. http://dx.doi.org/10.1001/archinte.164.19.2169

[25] Sorrentino, P., Tarantino, G., Conca, P., et al. (2004) Silent Non-Alcoholic Fatty Liver Disease a Clinical-Histological Study. Journal of Hepatology, 41, 751-757. http://dx.doi.org/10.1016/j.jhep.2004.07.010

[26] Amarapurkar, D., Kamani, P., Patel, N., et al. (2007) Prevalence of Non-Alcoholic Fatty Liver Disease: Population Based Study. Annals of Hepatology, 6, 161-163.

[27] Duseja, A., Das, A., Das, R., et al. (2007) The Clinicopathological Profile of Indian Patients with Nonalcoholic Fatty Liver Disease (NAFLD) Is Different from That in the West. Digestive Diseases and Sciences, 52, 2368-2374. http://dx.doi.org/10.1007/s10620-006-9136-y

[28] Dixon, J.B., Bhathal, P.S. and O’Brian, P.E. (2001) Non-Alcoholic Fatty Liver Disease: Predictors of Non-Alcoholic Steatohepatitis and Liver Fibrosis in the Severely Obese. Gastroenterology, 121, 91-100. http://dx.doi.org/10.1053/gast.2001.25540

[29] Marchesini, G., Bugianesi, E., Forlani, G., et al. (2003) Non Alcoholic Fatty Liver, Steatohepatitis, and the Metabolic Syndrome. Hepatology, 37, 917-923. http://dx.doi.org/10.1053/jhep.2003.50161

[30] Harnois, F., Msika, S., Sabaté, J.M., et al. (2006) Prevalence and Predictive Factors of NASH in Morbidly Obese Patients Undergoing Bariatric Surgery. Obesity Surgery, 16, 183-188. http://dx.doi.org/10.1381/096089206775565122

[31] Caldwell, S.H. and Argo, C.K. (2011) Non-Alcoholic Fatty Liver Disease and Nutrition. In: Sherlock's Diseases of the Liver and Biliary System, 12th Edition, 28, 546-561. http://dx.doi.org/10.1002/9781444341294.ch28

[32] Valenti, L., Al-Serri, A., Daly, A.K., et al. (2010) Homozygosity for the Patatin-Like Phospholipase-3/Adiponutrin I148 M Polymorphism Influences Liver Fibrosis in Patients with Nonalcoholic Fatty Liver Disease. Hepatology, 51, 1209-1217.

[33] Goran, M.I., Walker, R., Le, K.A., et al. (2010) Effects of PNPLA3 on Liver Fat and Metabolic Profile in Hispanic Children and Adolescents. Diabetes, 59, 3127-3130. http://dx.doi.org/10.2337/db10-0554

[34] Li, Y., Xing, C., Tian, Z. and Ku, H.C. (2012) Genetic Variant I148M in PNPLA3 Is Associated with the Ultrasonography-Determined Steatosis Degree in a Chinese Population. BMC Medical Genetics, 13, 113. http://dx.doi.org/10.1186/1471-2350-13-113

[35] Xu, R., Tao, A., Zhang, S., Deng, Y. and Chen, G. (2015) Association between Patatin-Like Phospholipase Domain Containing 3 Gene (PNPLA3) Polymorphisms and Nonalcoholic Fatty Liver Disease: A HuGE Review and MetaAnalysis. Scientific Reports, 5, 9284. http://dx.doi.org/10.1038/srep09284

[36] Zain, S.M. and Mohamed, R. (2012) A Multi-Ethnic Study of a PNPLA3 Gene Variant and Its Association with Disease Severity in Non-Alcoholic Fatty Liver Disease. Human Genetics, 131, 1145-1152. http://dx.doi.org/10.1007/s00439-012-1141-y

[37] Hotta, K., Yoneda, M., Hyogo, H., et al. (2010) Association of the rs738409 Polymorphism in PNPLA3 with Liver Damage and the Development of Nonalcoholic Fatty Liver Disease. BMC Medical Genetics, 11, 172. http://dx.doi.org/10.1186/1471-2350-11-172

[38] Lin, Y.C., Chang, P.F., Hu, F.C., Yang, W.S., Chang, M.H. and Ni, Y.H. (2011) A Common Variant in the PNPLA3 Gene Is a Risk Factor for Non-Alcoholic Fatty Liver Disease in Obese Taiwanese Children. The Journal of Pediatrics, 158, 740-744. http://dx.doi.org/10.1016/j.jpeds.2010.11.016

[39] Romeo, S., Sentinelli, F., Cambuli, V.M., et al. (2010) The 148M Allele of the PNPLA3 Gene Is Associated with Indices of Liver Damage Early in Life. Journal of Hepatology, 53, 335-338. http://dx.doi.org/10.1016/j.jhep.2010.02.034

[40] Valenti, A., Alisi, E., Galmozzi, A., et al. (2010) I148M Patatin-Like Phospholipase Domain Containing 3 Gene Variant and Severity of Pediatric Nonalcoholic Fatty Liver Disease. Hepatology, 52, 1274-1280.

[41] Speliotes, E.K., Butler, J.L., Palmer, C.D., Voight, B.F. and Hirschhorn, J.N. (2010) PNPLA3 Variants Specifically Confer Increased Risk for Histologic Nonalcoholic Fatty Liver Disease But Not Metabolic Disease. Hepatology, 52, 904-912. http://dx.doi.org/10.1002/hep.23768

[42] Jou, J., Choi, S.S. and Diehl, A.M. (2008) Mechanisms of Disease Progression in Nonalcoholic Fatty Liver Disease. Seminars in Liver Disease, 28, 370-379. http://dx.doi.org/10.1055/s-0028-1091981

[43] Valenti, L., Fracanzani, A.L., Dongiovanni, P., et al. (2002) Tumor Necrosis Factor Alpha Promoter Polymorphisms and Insulin Resistance in Nonalcoholic Fatty Liver Disease. Gastroenterology, 122, 274-280. http://dx.doi.org/10.1053/gast.2002.31065

[44] Tokushige, K., Takakura, M., Tsuchiya-Matsushita, N., et al. (2007) Influence of TNF Gene Polymorphisms in Japanese Patients with NASH and Simple Steatosis. Journal of Hepatology, 46, 1104-1110. http://dx.doi.org/10.1016/j.jhep.2007.01.028 
[45] Yang, H.R., Ko, J.S. and Seo, J.K. (2012) Role of Tumor Necrosis Factor- $\alpha$ Promoter Polymorphism and Insulin Resistance in the Development of Non-Alcoholic Fatty Liver Disease in Obese Children Pediatric Gastroenterology. Hepatology \& Nutrition, 15, 44-51.

[46] Chowdhury, S.D., Ramakrishna, B., Eapen, C.E., et al. (2013) Fibrosis in Non-Alcoholic Fatty Liver Disease: Correlation with Simple Blood Indices and Association with Tumor Necrosis Factor-Alpha Polymorphisms. Tropical Gastroenterology, 34, 31-35. http://dx.doi.org/10.7869/tg.2012.88

[47] Trujillo-Murillo, K., Bosques-Padilla, F.J., Calderón-Lozano, I., et al. (2011) Association of Tumor Necrosis Factor $\alpha$ and Manganese Superoxide Dismutase Polymorphisms in Patients with Non-Alcoholic Steatohepatitis from Northeast Mexico. The Open Hepatology Journal, 3, 1-6. http://dx.doi.org/10.2174/1876517301103010001

[48] Cheng, Y., An, B., Jiang, M., Xin, Y. and Xuan, S. (2015) Association of Tumor Necrosis Factor-Alpha Polymorphisms and Risk of Coronary Artery Disease in Patients with Non-Alcoholic Fatty Liver Disease. Hepatology, 15, e26818. http://dx.doi.org/10.5812/hepatmon.26818 